\title{
BERGMAN OPERATORS FOR PARABOLIC EQUATIONS IN TWO SPACE VARIABLES ${ }^{1}$
}

\author{
DAVID COLTON
}

\begin{abstract}
An integral operator is constructed which maps analytic functions of two complex variables onto the class of real valued analytic solutions of linear second order parabolic equations in two space variables with real valued, analytic, time independent coefficients. When the solution of the parabolic equation is independent of the time variable the operator reduces to Bergman's integral operator for elliptic equations in two independent variables.
\end{abstract}

I. Introduction. Although the analytic theory of elliptic equations has been extensively investigated by many mathematicians (cf. the monographs [1], [4], [8]), little has been done in developing an analogous theory for parabolic equations (however see [2], [5], [6]). An important method in the investigation of the analytic behaviour of solutions to elliptic equations has been the use of a variety of integral operators which map analytic functions onto solutions of the elliptic equation. In order to undertake a similar study of parabolic equations it would be desirable to have similar tools at our disposal. An initial step in this direction was taken by Bergman in [2] (see also [1, pp. 74-78]) who constructed an integral operator for certain classes of parabolic equations in two space variables. However in addition to having a very complicated structure and being applicable to only a limited class of equations, the operator constructed by Bergman is not an onto mapping. In particular Bergman's operator maps analytic functions into a subclass of solutions of the differential equation which have a Taylor expansion of a certain form. In this note we will overcome the difficulties inherent in Bergman's approach and construct an integral operator which maps analytic functions of two complex variables onto real valued analytic solutions of the general linear second order parabolic equation in two independent variables with real valued, analytic, time independent coefficients. (Our analysis can easily be modified to include the case in which the coefficients also depend on time.) In particular we

Received by the editors June 12, 1972.

AMS (MOS) subject classifications (1970). Primary 35C15, 35A20, 35K10.

Key words and phrases. Integral operator, Bergman operator, parabolic equation, elliptic equation, Goursat data, analytic function.

${ }^{1}$ This research was supported in part by NSF Grant GP-27232 and in part by the Science Research Council while the author was a visiting research fellow at the University of Glasgow.

(c) American Mathematical Society 1973 
will consider the parabolic equation (written in normal form)

$$
u_{x x}+u_{y y}+a(x, y) u_{x}+b(x, y) u_{y}+c(x, y) u=d(x, y) u_{t}
$$

and make the assumption that the coefficients of equation (1.1) are entire functions of their independent (complex) variables (with minor modifications we could have assumed only that these coefficients are analytic in some polydisc in the space of two complex variables) and are real valued for $x$ and $y$ real. When the solution of equation (1.1) is independent of $t$ we will show that our operator reduces to that of Bergman for elliptic equations in two independent variables.

An alternate method to that of Bergman for constructing integral operators for elliptic equations in two independent variables has been given by Vekua [8]. In [6] Hill has constructed an integral operator for parabolic equations which is analogous to that of Vekua for elliptic equations. The advantages (and disadvantages) of our operator in comparison with that of Hill are comparable to a similar comparison between the operators of Bergman and Vekua for elliptic equations (cf. [1, p. 2]). We will not enter into such a discussion at this time, except to point out that the kernel of our operator is considerably easier to construct than that of Hill since the kernel of Hill's operator is expressed as an infinite series, each of whose terms is computed by solving a complex Goursat problem for an elliptic equation in two independent variables.

II. An integral operator for equation (1.1). We first define the nonsingular transformation of the space $C^{2}$ of two complex variables into itself by

$$
z=x+i y, \quad z^{*}=x-i y .
$$

Under such a transformation equation (1.1) assumes the form

$$
U_{z z^{*}}+A\left(z, z^{*}\right) U_{z}+B\left(z, z^{*}\right) U_{z^{*}}+C\left(z, z^{*}\right) U=D\left(z, z^{*}\right) U_{t}
$$

where

$$
\begin{aligned}
U\left(z, z^{*}, t\right) & =u\left(\frac{z+z^{*}}{2}, \frac{z-z^{*}}{2 i}, t\right), \\
A\left(z, z^{*}\right) & =\frac{1}{4}\left[a\left(\frac{z+z^{*}}{2}, \frac{z-z^{*}}{2 i}\right)+i b\left(\frac{z+z^{*}}{2}, \frac{z-z^{*}}{2 i}\right)\right], \\
B\left(z, z^{*}\right) & =\frac{1}{4}\left[a\left(\frac{z+z^{*}}{2}, \frac{z-z^{*}}{2 i}\right)-i b\left(\frac{z+z^{*}}{2}, \frac{z-z^{*}}{2 i}\right)\right], \\
C\left(z, z^{*}\right) & =\frac{1}{4} c\left(\frac{z+z^{*}}{2}, \frac{z-z^{*}}{2 i}\right) \\
D\left(z, z^{*}\right) & =\frac{1}{4} d\left(\frac{z+z^{*}}{2}, \frac{z-z^{*}}{2 i}\right) .
\end{aligned}
$$


Setting

$$
V\left(z, z^{*}, t\right)=U\left(z, z^{*}, t\right) \exp \left\{\int_{0}^{z^{*}} A\left(z, \zeta^{*}\right) d \zeta^{*}\right\}
$$

reduces equation (2.2) to the canonical form

$$
V_{z z^{*}}+\widetilde{B}\left(z, z^{*}\right) V_{z^{*}}+\widetilde{C}\left(z, z^{*}\right) V=\tilde{D}\left(z, z^{*}\right) V_{t}
$$

where

$$
\begin{aligned}
& \widetilde{B}\left(z, z^{*}\right)=B\left(z, z^{*}\right)-\int_{0}^{z^{*}} A_{z}\left(z, \zeta^{*}\right) d \zeta^{*} \\
& \widetilde{C}\left(z, z^{*}\right)=-\left(A_{z}+A B-C\right) \\
& \tilde{D}\left(z, z^{*}\right)=D\left(z, z^{*}\right) .
\end{aligned}
$$

We now proceed to construct an integral operator which maps analytic functions of two complex variables onto analytic solutions of equation (2.5). In particular we look for solutions of equation (2.5) in the form

$$
\begin{aligned}
& V\left(z, z^{*}, t\right) \\
& \quad=\frac{-1}{2 \pi i} \oint_{|t-\tau|=\delta} \int_{-1}^{+1} E\left(z, z^{*}, t-\tau, s\right) f\left(\frac{z}{2}\left(1-s^{2}\right), \tau\right) \frac{d s d \tau}{\left(1-s^{2}\right)^{1 / 2}}
\end{aligned}
$$

where $\delta>0, f(z, t)$ is an analytic function of two complex variables in a neighborhood of the origin in $C^{2}$, and $E\left(z, z^{*}, t, s\right)$ is a function to be determined. The first integral in equation (2.7) is an integration in the complex $\tau$ plane in a counterclockwise direction about a circle of radius $\delta$ with centre at $t$, and the second integral is an integration over a curvilinear path in the unit disc in the complex $s$ plane joining the points $s=+1$ and $s=-1$. Substituting equation (2.7) into equation (2.5) and integrating by parts (cf. [1, p. 11]) show that $E\left(z, z^{*}, t, s\right)$ must satisfy the differential equation

$$
\left(1-s^{2}\right) E_{z^{*} s}-(1 / s) E_{z^{*}}+2 s z\left(E_{z z^{*}}+\widetilde{B} E_{z^{*}}+\widetilde{C} E-\tilde{D} E_{t}\right)=0,
$$

provided we also assume that $E\left(z, z^{*}, t, s\right)$ is an analytic function of $s$ for $|s| \leqq 1, t$ for $\delta_{0} \leqq|t| \leqq \delta_{1}$ (where $\left.\delta_{0}<\delta<\delta_{1}\right)$, and $\left(z, z^{*}\right)$ in some neighbourhood of the origin in $C^{2}$. Motivated by Bergman's analysis for elliptic equations in two independent variables we now look for a solution of equation (2.8) in the form

$$
E\left(z, z^{*}, t, s\right)=\frac{1}{t}+\sum_{n=1}^{\infty} s^{2 n} z^{n} \int_{0}^{z^{*}} P^{(2 n)}\left(z, \zeta^{*}, t\right) d \zeta^{*} .
$$

Substituting equation (2.9) into equation (2.8) yields the following 
recursion formula for the coefficients $P^{(2 n)}$ :

$$
P^{(2)}=-\frac{2}{t} \tilde{C}-\frac{2}{t^{2}} \tilde{D}
$$

$$
(2 n+1) P^{(2 n+2)}
$$

$$
\begin{array}{r}
=-2\left[P_{z}^{(2 n)}+\tilde{B} P^{(2 n)}+\widetilde{C} \int_{0}^{z^{*}} P^{(2 n)} d \zeta^{*}-\tilde{D} \int_{0}^{z^{*}} P_{t}^{(2 n)} d \zeta^{*}\right], \\
n=1,2, \cdots .
\end{array}
$$

Setting $P^{(2 n)}\left(z, z^{*}, t\right)=t^{-n-1} Q^{(2 n)}\left(z, z^{*}, t\right)$ in equation (2.10) yields the following recursion formula for the $Q^{(2 n)}$ :

$$
Q^{(2)}=-2 t \tilde{C}-2 \tilde{D},
$$

$(2 n+1) Q^{(2 n+2)}$

$$
\begin{aligned}
=-2\left[t Q_{z}^{(2 n)}+t \tilde{B} Q^{(2 n)}+t \tilde{C} \int_{0}^{z^{*}} Q^{(2 n)} d \zeta^{*}\right. \\
\left.+(n+1) \tilde{D} \int_{0}^{z^{*}} Q^{(2 n)} d \zeta^{*}-t \tilde{D} \int_{0}^{z^{*}} Q_{t}^{(2 n)} d \zeta^{*}\right], \\
n=1,2, \cdots .
\end{aligned}
$$

It is clear from equation (2.10) that each of the $P^{(2 n)}, n=1,2, \cdots$, is uniquely determined. In order to show the existence of the function $E\left(z, z^{*}, t, s\right)$ it is now necessary to show the convergence of the series (2.9). To this end we first majorize the functions $Q^{(2 n)}\left(z, z^{*}, t\right)$. Let $r$ be an arbitrarily large positive number and let $B_{0}$ be a positive constant chosen such that for $|z|<r,\left|z^{*}\right|<r$, we have

$$
\begin{aligned}
& \widetilde{B}\left(z, z^{*}\right) \ll \frac{B_{0}}{(1-z / r)\left(1-z^{*} / r\right)}, \\
& \widetilde{C}\left(z, z^{*}\right) \ll \frac{B_{0}}{(1-z / r)\left(1-z^{*} / r\right)}, \\
& \tilde{D}\left(z, z^{*}\right) \ll \frac{B_{0}}{(1-z / r)\left(1-z^{*} / r\right)},
\end{aligned}
$$

where "«" denotes domination (cf. [1], [4]). We will now show by induction that there exist positive constants $M_{n}$ and $\varepsilon$ (where $\varepsilon$ is independent of $n$ and $M_{n}$ is a bounded function of $n$ ) such that for $|z|<r$, 
$\left|z^{*}\right|<r,|t|<\delta_{1}$, we have

$$
\begin{aligned}
Q^{(2 n)} \ll & \frac{M_{n} 2^{2 n} \delta_{1}^{n}(1+\varepsilon)^{n}}{2 n-1} \\
& \times\left(1-\frac{z}{r}\right)^{-(2 n-1)}\left(1-\frac{z^{*}}{r}\right)^{-(2 n-1)}\left(1-\frac{t}{2 \delta_{1}}\right)^{-(2 n-1)} r^{-n}
\end{aligned}
$$

This is clearly true for $n=1$. Now suppose for $n=k$ equation (2.13) is valid. Then from equations (2.11) and (2.12) and the straightforward use of the theory of dominants we have

$$
\begin{aligned}
Q^{(2 k+2)} \ll & \frac{M_{k} 2^{2 k+2} \delta_{1}^{k+1}(1+\varepsilon)^{k}}{2 k+1} \\
& \times\left\{1+\frac{B_{0}}{2 k-1}\left(r+\frac{r^{2}}{(2 k-1)}+\frac{r^{2}(k+1)}{(2 k-1) 2 \delta_{1}}+\frac{r^{2}}{2 \delta_{1}}\right)\right\} \\
& \times\left(1-\frac{z^{*}}{r}\right)^{-(2 k+1)}\left(1-\frac{z}{r}\right)^{-(2 k+1)}\left(1-\frac{t}{2 \delta_{1}}\right)^{-(2 k+1)} r^{-k-1} .
\end{aligned}
$$

In the derivation of equation (2.14) we have made use of the fact that $t \ll 2 \delta_{1}\left(1-t / 2 \delta_{1}\right)^{-1}$ and that if $f \ll g$ then

$$
f \ll g(1-z / r)^{-j}\left(1-z^{*} / r\right)^{-k}\left(1-t / 2 \delta_{1}\right)^{-l}
$$

for arbitrary positive integers $j, k$, and $l$.

By setting

$$
M_{n+1}=M_{n}(1+\varepsilon)^{-1}\left\{1+\frac{B_{0} r}{(2 n-1)^{2}}\left(2 n-1+r+\frac{3 n r}{2 \delta_{1}}\right)\right\}
$$

we have shown that equation (2.13) is true for $n=k+1$, thus completing the induction step. Note that for $n$ sufficiently large we have $M_{n+1} \leqq M_{n}$, i.e. there exists a positive constant $M$ which is independent of $n$ such that $M_{n} \leqq M$ for all $n$.

We now turn to the convergence of the series (2.9). Let $s_{0} \geqq 1$ and $\alpha>1$ be positive constants and let $|s| \leqq s_{0},|z|<r / \alpha,\left|z^{*}\right|<r / \alpha$, and $\delta_{0} \leqq|t| \leqq \delta_{1}$. Then $(1-|z| \mid r) \geqq(\alpha-1) / \alpha, \quad\left(1-\left|z^{*}\right| / r\right) \geqq(\alpha-1) / \alpha, \quad\left(1-|t| / 2 \delta_{1}\right) \geqq \frac{1}{2}$, and from equation (2.14) it is seen that the series (2.9) is majorized by the series

$$
\frac{1}{\delta_{0}}+\sum_{n=1}^{\infty} \frac{r M_{n} 2^{4 n-1} s_{0}^{2 n} \delta_{1}^{n}(1+\varepsilon)^{n} \alpha^{3 n-2}}{\delta_{0}^{n+1}(2 n-1)(\alpha-1)^{4 n-2}} .
$$

If $\alpha$ is chosen such that $16 s_{0}^{2} \delta_{1}(1+\varepsilon) \alpha^{3} \delta_{0}^{-1}(\alpha-1)^{-4}<1$, then the series (2.16) is convergent. Since $r$ is an arbitrarily large positive number and $\varepsilon$ is arbitrarily small and independent of $r$, we can now conclude that the series (2.9) converges absolutely and uniformly for $|z|<r,\left|z^{*}\right|<r,|s| \leqq s_{0}$, 
$\delta_{0} \leqq|t| \leqq \delta_{1}$ for $r, \delta_{1}$, and $s_{0}$ arbitrarily large and $\delta_{0}>0$ arbitrarily small, i.e. $E\left(z, z^{*}, t, s\right)$ is an entire function of its independent variables except for an (essential) singularity at $t=0$.

We have now shown that the operator $\boldsymbol{P}_{2}$ defined by

$$
U\left(z, z^{*}, t\right)=\boldsymbol{P}_{2}\{f\}
$$

$$
\begin{aligned}
= & \frac{-1}{2 \pi i} \exp \left\{-\int_{0}^{z^{*}} A\left(z, \zeta^{*}\right) d \zeta^{*}\right\} \\
& \times \oint_{|t-\tau|=\delta} \int_{-1}^{+1} E\left(z, z^{*}, t-\tau, s\right) f\left(\frac{z}{2}\left(1-s^{2}\right) \tau\right) \frac{d s d \tau}{\left(1-s^{2}\right)^{1 / 2}}
\end{aligned}
$$

exists and maps analytic functions which are regular in some neighbourhood of the origin in $C^{2}$ into the class of (complex valued) solutions of equation (2.2). An elementary power series analysis (cf. [8, pp. 55-56]) coupled with Hormander's generalized Cauchy-Kowalewski theorem [7] shows that solutions of equation (2.2) which are real valued for $t$ real and $z^{*}=\bar{z}$ (i.e. $x$ and $y$ real) are uniquely determined by their values on the characteristic plane $z^{*}=0$. Furthermore, since the coefficients of equation (1.1) are real valued for $x$ and $y$ real, the operator $\operatorname{Re} \boldsymbol{P}_{2}\{f\}$ (where "Re" denotes "take the real part") defines a real valued solution of equation (1.1) provided we set $z^{*}=\bar{z}$ and keep $t$ real. Evaluating $\operatorname{Re} \boldsymbol{P}_{2}\{f\}$ at $z^{*}=0$ and keeping $t$ real gives

$$
\begin{array}{r}
U(z, 0, t)=\frac{-1}{4 \pi i} \oint_{|t-\tau|=\delta} \int_{-1}^{+1}\left[f\left(\frac{z}{2}\left(1-s^{2}\right), \tau\right)\right. \\
\left.+\bar{f}(0, \tau) \exp \left(-\int_{0}^{z} \bar{A}\left(0, \zeta^{*}\right) d \zeta^{*}\right)\right] \\
\times \frac{d s d \tau}{\left(1-s^{2}\right)^{1 / 2}(t-\tau)} \\
=\frac{1}{2} \int_{-1}^{+1} f\left(\frac{z}{2}\left(1-s^{2}\right), t\right) \frac{d s}{\left(1-s^{2}\right)^{1 / 2}}+\frac{\pi}{2} \bar{f}(0, t) \\
\times \exp \left(-\int_{0}^{z} \bar{A}\left(0, \zeta^{*}\right) d \zeta^{*}\right)
\end{array}
$$

where

$$
\bar{f}(z, t)=\overline{f(\bar{z}, t)} \text { and } \bar{A}\left(z, z^{*}\right)=\overline{A\left(\bar{z}, \bar{z}^{*}\right)} .
$$

A solution of the integral equation (2.18) is given by [1, p. 12]

$$
\begin{aligned}
& f\left(\frac{z}{2}, t\right)=-\frac{1}{2 \pi} \int_{\gamma}\left[2 U\left(z\left(1-s^{2}\right), 0, t\right)\right. \\
&\left.U(0,0, t) \exp \left(-\int_{0}^{z} \bar{A}\left(0, \zeta^{*}\right) d \zeta^{*}\right)\right] \frac{d s}{s^{2}}
\end{aligned}
$$


where $\gamma$ is a rectifiable arc joining the points $s=-1$ and $s=+1$ and not passing through the origin. Equations (2.18) and (2.19) show that if $U(z, \bar{z}, t)$ is real valued for $t$ real, then $f(z, t)$ can be chosen such that $U(z, 0, t)$ assumes prescribed values. We thus have the following theorem:

THEOREM. Let $u(x, y, t)$ be a real valued analytic solution of equation (1.1) defined in some neighborhood of the origin. Then $u(x, y, t)=U(z, \bar{z}, t)$ can be represented in the form

$$
\begin{aligned}
U(z, \bar{z}, t)= & \operatorname{Re} \boldsymbol{P}_{2}\{f\} \\
= & \operatorname{Re}\left[\frac{-1}{2 \pi i} \exp \left\{-\int_{0}^{z} A\left(z, \zeta^{*}\right) d \zeta^{*}\right\}\right. \\
& \left.\cdot \oint_{|t-\tau|=\delta} \int_{-1}^{+1} E(z, \bar{z}, t-\tau, s) f\left(\frac{z}{2}\left(1-s^{2}\right), \tau\right) \frac{d s d \tau}{\left(1-s^{2}\right)^{1 / 2}}\right]
\end{aligned}
$$

where $E\left(z, z^{*}, t, s\right)$ is defined by equations (2.9) and (2.10) and is an entire function of its independent variables except for an essential singularity at $t=0$, and $f(z, t)$ is defined by equation (2.19) and is analytic in some neighborhood of the origin in $C^{2}$. Conversely, for every analytic function $f(z, t)$ defined in some neighbourhood of the origin in $C^{2}$, equation (2.20) defines a real valued analytic solution of equation (1.1) in some neighbourhood of the origin.

The representation (2.20) can now be used to analytically continue solutions of parabolic equations. For the type of theorems which can be obtained the reader is referred to the results for elliptic equations in two independent variables obtained in [1]. The operator defined by equation (2.20) is in fact closely related to Bergman's operator for elliptic equations in two independent variables. To see this we consider the case in which $u(x, y, t)=u(x, y)$ is independent of $t$ and hence satisfies the elliptic equation

$$
u_{x x}+u_{y y}+a(x, y) u_{x}+b(x, y) u_{y}+c(x, y) u=0 .
$$

In this situation the associated analytic function $f(z, t)=f(z)$ is independent of $t$, and termwise integration in equation (2.20) yields the representation

$$
\begin{aligned}
U(z, \bar{z})=\operatorname{Re}\left[\exp \left\{-\int_{0}^{i} A\left(z, \zeta^{*}\right) d \zeta^{*}\right\}\right. & \\
& \times \int_{-1}^{+1} E(z, \bar{z}, s) f\left(\frac{z}{2}\left(1-s^{2}\right)\right) \frac{d s}{\left(1-s^{2}\right)^{1 / 2}}
\end{aligned}
$$


where

$$
E\left(z, z^{*}, s\right)=1+\sum_{n=1}^{\infty} s^{2 n} z^{n} \int_{0}^{z^{*}} P^{(2 n)}\left(z, \zeta^{*}\right) d \zeta^{*}
$$

with the $P^{(2 n)}$ being defined recursively by

$$
\begin{aligned}
& P^{(2)}=-2 \widetilde{C} \\
&(2 n+1) P^{(2 n+2)}=-2\left[P_{z}^{(2 n)}+\widetilde{B} P^{(2 n)}+\widetilde{C} \int_{0}^{z^{*}} P^{(2 n)} d \zeta^{*}\right] \\
& n \\
& n=1,2, \cdots
\end{aligned}
$$

A comparison of equations (2.22)-(2.24) with the corresponding formula in [1] shows that the operator defined by equation (2.22) is identical with Bergman's operator for elliptic equations in two independent variables.

In closing we note that it is also of interest to compare our integral representation (2.20) for parabolic equations in two space variables with the corresponding representation for elliptic equations in three independent variables obtained in [3].

\section{REFERENCES}

1. S. Bergman, Integral operators in the theory of linear partial differential equations, Ergebnisse der Mathematik und ihrer Grenzgebiete, N.F., Heft 23, Springer-Verlag, Berlin, 1961, pp. 207-222. MR 25 \#213.

2. - On singularities of solutions of certain differential equations in three variables, Trans. Amer. Math. Soc. 85 (1957), 462-488. MR 19, 654.

3. D. Colton, Bergman operators for elliptic equations in three independent variables, Bull. Amer. Math. Soc. 77 (1971), 752-756. MR 43 \#6578.

4. R. P. Gilbert, Function theoretic methods in partial differential equations, Math. in Sci. and Engineering, vol. 54, Academic Press, New York, 1969. MR 39 \#3127.

5. C. D. Hill, Parabolic equations in one space variable and the non-characteristic Cauchy problem, Comm. Pure Appl. Math. 20 (1967), 619-633. MR 35 \#5774.

6. - A method for the construction of reflection laws for a parabolic equation, Trans. Amer. Math. Soc. 133 (1968), 357-372. MR 38 \#3597.

7. L. Hörmander, Linear partial differential operators, 3rd rev. ed., Die Grundlehren der math. Wissenschaften, Band 116, Springer-Verlag, New York, 1969. MR 40 \#1687.

8. I. N. Vekua, New methods for solving elliptic equations, OGIZ, Moscow, 1948; English transl., Series in Appl. Math., vol. 1, North-Holland, Amsterdam; Interscience, New York, 1967. MR 11, 598; MR 35 \#3243.

Department of Mathematics, Indiana University, Bloomington, Indiana 47401

Department of Mathematics, University of Glasgow, Glasgow, Scotland 